\title{
Minimum Length from Quantum Mechanics and Classical General Relativity
}

\author{
Xavier Calmet, ${ }^{*}$ Michael Graesser, ${ }^{\dagger}$ and Stephen D. $\mathrm{H} \mathrm{Hsu}^{\ddagger}$ \\ California Institute of Technology, Pasadena, California 91125, USA
}

(Received 7 May 2004; published 15 November 2004)

\begin{abstract}
We derive fundamental limits on measurements of position, arising from quantum mechanics and classical general relativity. First, we show that any primitive probe or target used in an experiment must be larger than the Planck length $l_{P}$. This suggests a Planck-size minimum ball of uncertainty in any measurement. Next, we study interferometers (such as LIGO) whose precision is much finer than the size of any individual components and hence are not obviously limited by the minimum ball. Nevertheless, we deduce a fundamental limit on their accuracy of order $l_{P}$. Our results imply a device independent limit on possible position measurements.
\end{abstract}

DOI: 10.1103/PhysRevLett.93.211101

PACS numbers: 04.20.-q, 03.65.-w

It is widely believed that a minimum length, of order the Planck length $l_{P}$, results from combining quantum mechanics and classical general relativity [1-3]. That is, no operational procedure (experiment) exists which can measure a distance less than of order $l_{P}$. The key ingredients used to reach this conclusion are the uncertainty principle from quantum mechanics, and gravitational collapse (black hole formation) from general relativity.

A dynamical condition for gravitational collapse is given by the Hoop Conjecture (HC) [4], due to Kip Thorne: if an amount of energy $E$ is confined at any instant to a ball of size $R$, where $R<E$, then that region will eventually evolve into a black hole [5]. Recent results on black hole production in particle collisions [6] show strong support for the $\mathrm{HC}$, even in the least favorable instance where all of the energy $E$ is in the kinetic energy of two particles moving past each other at the speed of light.

From the $\mathrm{HC}$ and the uncertainty principle, we immediately deduce the existence of a minimum ball of size $l_{P}$. Consider a particle of energy $E$ which is not already a black hole. Its size $r$ must satisfy

$$
r \gtrsim \max [1 / E, E],
$$

where $\lambda_{C} \sim 1 / E$ is its Compton wavelength and $E$ arises from the hoop conjecture. Minimization with respect to $E$ results in $r$ of order unity in Planck units [7], or $r \sim l_{P}$. If the particle is a black hole, then its radius grows with mass: $r \sim E \sim 1 / \lambda_{C}$. This relationship suggests that an experiment designed (in the absence of gravity) to measure a short distance $l \ll l_{P}$ will (in the presence of gravity) only be sensitive to distances $1 / l$. This is the classical counterpart to $T$ duality in string theory [9].

It is possible that quantum gravitational corrections modify the relation between $E$ and $R$ in the HC [10]. However, if $E$ is much larger than the Planck mass, and $R$ much larger than $l_{P}$, we expect semiclassical considerations to be reliable. (Indeed, in two particle collisions with center-of mass energy much larger than the Planck mass the black holes produced are semiclassical.) This means that the existence of a minimum ball of size much smaller than $l_{P}$ does not depend on quantum gravity - the energy required to confine a particle to a region of size much smaller than $l_{P}$ would produce a large, semiclassical black hole.

Before proceeding further, we give a concrete model of minimum length that will be useful later. Let the position operator $\hat{x}$ have discrete eigenvalues $\left\{x_{i}\right\}$, with the separation between eigenvalues either of order $l_{P}$ or smaller. (For regularly distributed eigenvalues with a constant separation, this would be equivalent to a spatial lattice.) We do not mean to imply that nature implements minimum length in this particular fashion-most likely, the physical mechanism is more complicated, and may involve, for example, spacetime foam or strings. However, our concrete formulation lends itself to detailed analysis. We show below that this formulation cannot be excluded by any gedanken experiment, which is strong evidence for the existence of a minimum length.

Quantization of position does not by itself imply quantization of momentum. Conversely, a continuous spectrum of momentum does not imply a continuous spectrum of position. In a formulation of quantum mechanics on a regular spatial lattice, with spacing $a$ and size $L$, the momentum operator has eigenvalues which are spaced by $1 / L$. In the infinite volume limit the momentum operator can have continuous eigenvalues even if the spatial lattice spacing is kept fixed. This means that the displacement operator

$$
\hat{x}(t)-\hat{x}(0)=\hat{p}(0) \frac{t}{M}
$$

does not necessarily have discrete eigenvalues [the righthand side of (2) assumes free evolution; we use the Heisenberg picture throughout]. Since the time evolution operator is unitary the eigenvalues of $\hat{x}(t)$ are the same as $\hat{x}(0)$. Importantly though, the spectrum of $\hat{x}(0)[$ or $\hat{x}(t)]$ is completely unrelated to the spectrum of the $\hat{p}(0)$, even though they are related by (2) [11]. Consequently, we 
stress that a measurement of the displacement is a measurement of the spectrum of $\hat{p}(0)$ (for free evolution) and does not provide information on the spectrum of $\hat{x}$. A measurement of arbitrarily small displacement (2) does not exclude our model of minimum length. To exclude it, one would have to measure a position eigenvalue $x$ and a nearby eigenvalue $x^{\prime}$, with $\left|x-x^{\prime}\right| \ll l_{P}$.

Many minimum length arguments (involving, e.g., a microscope or scattering experiment [1]) are obviated by the simple observation of the minimum ball. However, the existence of a minimum ball does not by itself preclude the localization of a macroscopic object to very high precision. Hence, one might attempt to measure the spectrum of $\hat{x}(0)$ through a time of flight experiment in which wave packets of primitive probes are bounced off of welllocalized macroscopic objects. Disregarding gravitational effects, the discrete spectrum of $\hat{x}(0)$ is in principle obtainable this way. But, detecting the discreteness of $\hat{x}(0)$ requires wavelengths comparable to the eigenvalue spacing. For eigenvalue spacing comparable or smaller than $l_{P}$, gravitational effects cannot be ignored, because the process produces minimal balls (black holes) of size $l_{P}$ or larger. This suggests a direct measurement of the position spectrum to accuracy better than $l_{P}$ is not possible. The failure here is due to the use of probes with very short wavelength.

A different class of instrument - the interferometeris capable of measuring distances much smaller than the size of any of its subcomponents [12]. An interferometer can measure a distance

$$
\Delta x \sim \frac{\lambda}{b \sqrt{N}} \sim \frac{L}{\tau \sqrt{N} \nu},
$$

where $\lambda=1 / \nu$ is the wavelength of light used, $L$ is the length of each arm, $\tau$ the time duration of the measurement, and $N$ the number of photons. More precisely, $\Delta x$ is the change over the duration of the measurement in the relative path lengths of the two arms of the interferometer. $b=\tau / L$ is the number of bounces over which the phase difference builds, so (3) can also be written as

$$
\Delta \Phi=\frac{b \Delta x}{\lambda} \sim \frac{1}{\sqrt{N}},
$$

which expresses saturation of the quantum mechanical uncertainty relationship between the phase and number operators of a coherent state.

From (3) it appears that $\Delta x$ can be made arbitrarily small relative to $\lambda$ by, e.g., taking the number of bounces to infinity [13]. Were this the case, we would have an experiment that, while still using a wavelength $\lambda$ much larger than $l_{P}$, could measure a distance less than $l_{P}$ along one direction, albeit at the cost of making the measured object (e.g., a gravity wave) large in the time direction. This would contradict the existence of a minimum interval, though not a minimum ball in spacetime. (Another limit which increases the accuracy of the interferometer is to take the number of photons $N$ to infinity, but this is more directly constrained by gravitational collapse. Either limit is ultimately bounded by the argument discussed below.)

A constraint which prevents an arbitrarily accurate measurement of $\Delta x$ by an interferometer arises due to the standard quantum limit (SQL) and gravitational collapse. The SQL [14] is derived from the uncertainty principle (we give the derivation below; it is not specific to interferometers, although see [15]) and requires that

$$
\Delta x \geq \sqrt{\frac{t}{2 M}},
$$

where $t$ is the time over which the measurement occurs and $M$ the mass of the object whose position is measured. In order to push $\Delta x$ below $l_{P}$, we take $b$ and $t$ to be large. But from (5) this requires that $M$ be large as well. In order to avoid gravitational collapse, the size $R$ of our measuring device must also grow such that $R>M$. However, by causality $R$ cannot exceed $t$. Any component of the device a distance greater than $t$ away cannot affect the measurement, hence we should not consider it part of the device. These considerations can be summarized in the inequalities

$$
t>R>M \text {. }
$$

Combined with the SQL (5), they require $\Delta x>1$ in Planck units, or

$$
\Delta x>l_{P} .
$$

(Again, we neglect factors of order 1.)

Notice that the considerations leading to (5)-(7) were in no way specific to an interferometer, and hence are device independent. We repeat: no device subject to the SQL, gravity, and causality can exclude the quantization of position on distances less than the Planck length.

It is important to emphasize that we are deducing a minimum length which is parametrically of order $l_{P}$, but may be larger or smaller by a numerical factor. This point is relevant to the question of whether an experimenter might be able to transmit the result of the measurement before the formation of a closed trapped surface, which prevents the escape of any signal. If we decrease the minimum length by a numerical factor, the inequality (5) requires $M \gg R$, so we force the experimenter to work from deep inside an apparatus which has far exceeded the criteria for gravitational collapse (i.e., it is much denser than a black hole of the same size $R$ as the apparatus). For such an apparatus a horizon will already exist before the measurement begins. The radius of the horizon, which is of order $M$, is very large compared to $R$, so that no signal can escape.

We now give the derivation of the standard quantum limit. Consider the Heisenberg operators for position $\hat{x}(t)$ 
and momentum $\hat{p}(t)$ and recall the standard inequality

$$
(\Delta A)^{2}(\Delta B)^{2} \geq-\frac{1}{4}(\langle[\hat{A}, \hat{B}]\rangle)^{2} .
$$

Suppose that the position of a free test mass is measured at time $t=0$ and again at a later time. The position operator at a later time $t$ is

$$
\hat{x}(t)=\hat{x}(0)+\hat{p}(0) \frac{t}{M} .
$$

The commutator between the position operators at $t=0$ and $t$ is

$$
[\hat{x}(0), \hat{x}(t)]=i \frac{t}{M},
$$

so using (8) we have

$$
|\Delta x(0)||\Delta x(t)| \geq \frac{t}{2 M}
$$

We see that at least one of the uncertainties $\Delta x(0)$ or $\Delta x(t)$ must be larger than of order $\sqrt{t / M}$. As a measurement of the discreteness of $\hat{x}(0)$ requires two position measurements, it is limited by the greater of $\Delta x(0)$ or $\Delta x(t)$, that is, by $\sqrt{t / M}$.

The assumption of a free test mass in the SQL derivation deserves further scrutiny. One might imagine that specially designed interactions with the test mass during the time interval $(0, t)$ might alter the bound by extracting some of the momentum uncertainty. However, we now argue that if the mass $M$ is that of the entire experimental apparatus (as restricted by causality above), the SQL applies.

As a simple model for interactions between the test mass $m_{1}$ and the rest of the apparatus, imagine a spring connecting it to another mass $m_{2}$. If $m_{2} \gg m_{1}$ the spring damps out the uncertainty in the position of $m_{1}$ due to the position measurement at $t$. [The time evolution of $\hat{x}(t)$ would involve the harmonic oscillator potential, not just the free kinetic energy used to obtain (9).] We could further imagine that $m_{2}$ is connected to other masses $m_{i} \gg m_{2}$, etc. However, this construction terminates, due to causality, with any masses which are further than $t$ away from $m_{1}$ : they are not part of the experiment and can be neglected. Let the total mass of the system of masses and springs be $M \sim \sum m_{i}$. There is an uncertainty in the center-of mass coordinate $x_{\mathrm{cm}}$ of this system due to the measurement performed on $m_{1}$ at time $t=0$. Using causality, we can show that $\hat{x}_{\mathrm{cm}}(t)$ evolves freely as in (9) with $M$ given by the total mass: (a) anything outside the causal radius $t$ cannot affect the experiment, so we can simply remove it from our gedanken universe without changing the results, and (b) the position of an isolated apparatus in an empty universe must evolve freely according to (9). The uncertainty $\Delta x_{\mathrm{cm}}$ contributes to $\Delta x$, which one can see by writing $\hat{x}(t)=\hat{x}_{\mathrm{cm}}(t)+\hat{y}(t)$, with $\left[\hat{x}_{\mathrm{cm}}(t), \hat{y}(t)\right]=0$. We obtain a bound on $\Delta x$ which is independent of the specifics of the interactions-we need only use the total mass $M$ of all objects which can interact with $m_{1}$ during the measurement.

The argument of the previous paragraph focuses on the center-of-mass degree of freedom, but there are classes of experiments - such as interferometers - that are only sensitive to relative changes in position. For free particle motion the minimal length bound detailed above also applies to the relative coordinate. However, one might imagine interactions involving the relative degree of freedom that could limit the growth of uncertainty. Still, there are fundamental limits on the ability of an external potential to extract the uncertainty in momentum introduced by the initial measurement of $x(t)$. Let the Hamiltonian for each arm be that of a simple oscillator

$$
H=\frac{1}{2} m \dot{x}^{2}+\frac{1}{2} m \nu^{2}\left(x-x_{0}\right)^{2} .
$$

The width of the ground state wave function is $\sigma \sim$ $1 / \sqrt{m \nu}$, which must be less than $l_{P}$. In natural units, this requires $m \gg \nu^{-1}$. The $\mathrm{HC}$ then requires $L>m \gg$ $\nu^{-1}$, which contradicts the causality requirement that $L<\nu^{-1}$-i.e., that the size of the arm (spring plus masses) not exceed the oscillation time scale. If the causality requirement is violated, the system no longer behaves like an oscillator with a single displacement degree of freedom $x$.

There has been considerable discussion in the literature of defeating the SQL using contractive states $[16,17]$ or other quantum nondemolition (QND) techniques $[15,18-$ 21]. Contractive states allow for uncertainties in position that do not grow in time as rapidly as (5). Naively this may seem to allow for an accurate measurement of the discreteness of the position operator, but recall that two measurements of position are needed to do this. Straightforward algebra using the properties of contractive states (see, e.g., [16]) shows that the uncertainties in two subsequent measurements of position are still bounded by (11). Alternatively, this follows directly from the Heisenberg operator equations of motion, or more intuitively, because for a given level of desired uncertainty for both measurements, the time between measurements cannot be arbitrarily long, since for these states the uncertainty in position eventually begins to grow. We emphasize that as (11), not (5), was essential to our derivation, the use of contractive states will not allow for a measurement of the discreteness of position on scales less than the Planck scale.

Similarly, we note that the QND proposals typically amount to measurements of the displacement operator (2), or of the time-integrated force on a test mass, which would appear on the right-hand side of (2) if we had not assumed free evolution. In measuring the displacement operator [but not the position operator $\hat{x}(t)$ itself at different times], correlations between the initial and final states can be used to cancel the dependence on the initial state of 
the test mass. However, we have argued that such measurements do not probe the discretization of the position operator, and hence cannot address the question of minimum length.

The authors would like to thank C. Bauer, V. Braginsky, Y.Chen, M. Perelstein, J. Preskill, K. S. Thorne, A. Weinstein, and M. Wise for discussions. C. Bauer contributed to this work at an early stage. This research supported in part under DOE Contracts No. DE-FG0685ER40224 and No. DE-FG03-92ER40701.

*Email address: calmet@theory.caltech.edu

†Email address: graesser@theory.caltech.edu

${ }^{\ddagger}$ Permanent address: Institute of Theoretical Science and Department of Physics, University of OR, Eugene OR 97403.

Email address: hsu@duende.uoregon.edu

[1] L. J. Garay, Int. J. Mod. Phys. A 10, 145 (1995); C. A. Mead, Phys. Rev. 135, B849 (1964);T. Padmanabhan, Classical Quantum Gravity 4, L107 (1987).

[2] For earlier work using the quantum and gravitational limitations of a clock, seeJ. C. Baez and S. J. Olson, Classical Quantum Gravity 19, L121 (2002); Y.J. Ng and H. van Dam, Found. Phys. 30, 795 (2000); Phys. Lett. B 477, 429 (2000); G. Amelino-Camelia, Phys. Lett. B 477, 436 (2000); R. J. Adler, I. M. Nemenman, J. M. Overduin, and D. I. Santiago, Phys. Lett. B 477, 424 (2000).

[3] For a discussion of a minimum length in the context on quantum foam see, e.g., G. Amelino-Camelia, Nature (London) 398, 216 (1999); gr-qc/0104005; Y. J. Ng, gr-qc/ 0405078 .

[4] K. S. Thorne, in Magic Without Magic, edited by J. R. Klauder (W. H. Freeman, San Francisco, 1972), pp. 231258.

[5] We use natural units where $\hbar, c$, and Newton's constant (or $l_{P}$ ) are unity. We also neglect numerical factors of order 1.

[6] D. M. Eardley and S. B. Giddings, Phys. Rev. D 66, 044011 (2002); S. D. H. Hsu, Phys. Lett. B 555, 92 (2003).
[7] In this Letter we focus specifically on spacelike minimum intervals. By Lorentz covariance, we might expect that no spacetime interval $d s^{2}$ can be measured to accuracy better than $l_{P}^{2}$, but this is a more subtle issue [8].

[8] G. Amelino-Camelia, Int. J. Mod. Phys. D 11, 35 (2002); Phys. Lett. B 510, 255 (2001); J. Magueijo and L. Smolin, Phys. Rev. D 67, 044017 (2003).

[9] J. Polchinski, An Introduction to the Bosonic String, String Theory Vol. 1 (Cambridge University Press, Cambridge, 1998).

[10] V. P. Frolov and G. A. Vilkovisky, Phys. Lett. B 106, 307 (1981); Proceedings of the Second Seminar on Quantum Gravity, Moscow, 1981, edited by M. A. Markov and P. C. West (Plenum, London, 1983), p. 267.

[11] In general the discrete spectrum of an observable $A(0)$ is unrelated to that of $A(t)-A(0)$. For instance, consider a Hamiltonian $H=\alpha \sigma_{z}$ and $A(0)=\sigma_{x}$. Then the spectrum of $A(0)$ is $\pm 1 / 2$, but the spectrum of $\sigma_{x}(t)-\sigma_{x}(0)$ is $\pm 2 \sin (\alpha t / 2)$.

[12] http://www.ligo.caltech.edu/

[13] The parametrically improved sensitivity of the interferometer compared to the naive expectation $\Delta x \sim \lambda / b$ is achieved by monitoring the intensity of the recombined beams at the output port.

[14] V. B. Braginsky and Yu. I. Vorontsov, Usp. Fiz. Nauk 114, 41 (1974); Sov. Phys. Usp. 17, 644 (1975); C. M. Caves, Phys. Rev. Lett. 54, 2465 (1985).

[15] C. M. Caves, Phys. Rev. Lett. 45, 75 (1980); K. S. Thorne, R.W. P. Drever, C. M. Caves, M. Zimmermann, and V. D. Sandberg, Phys. Rev. Lett. 40, 667 (1978).

[16] H. P. Yuen, Phys. Rev. Lett. 51, 719 (1983); 52, 788 (1984).

[17] M. Ozawa, Phys. Rev. Lett. 60, 385 (1988).

[18] O. Alter and Y. Yamamoto, Phys. Lett. A 263, 226 (1999).

[19] V. B. Braginsky, M. L. Gorodetsky, and F. Ya. Khalili, Phys. Lett. A 232, 340 (1997); V. B. Braginsky and F. Ya. Khalili, Phys. Lett. A 257, 241 (1999).

[20] V. B. Braginsky, M. L. Gorodetsky, F. Y. Khalili, A. B. Matsko, K. S. Thorne, and S. P. Vyatchanin, Phys. Rev. D 67, 082001 (2003).

[21] A. Buonanno and Y. Chen, Classical Quantum Gravity 18, L95 (2001); Phys. Rev. D 64, 042006 (2001); 65 042001 (2002). 